\title{
Squamous cell carcinoma antigen as an adjunct tumour marker in primary carcinoma of the lung
}

\author{
P L Cheah, C K Liam, S F Yap, L M Looi
}

\begin{abstract}
Aims-To determine (1) the detection rate of primary carcinoma of the lung by serological assay of CEA (carcinoembryonic antigen); and (2) whether addition of seroassay of squamous cell carcinoma related antigen before treatment improves detection sensitivity.
\end{abstract}

Methods-A prospective study spanning 27 months was conducted at the University Hospital, Kuala Lumpur. Serum CEA (Abbott IMx) and serum squamous cell carcinoma antigen (Abbott IMx) from patients clinically suspected of having primary carcinoma of the lung, were assayed using the microparticle enzyme immunoassay method.

Results-Thirty seven cases of histologically confirmed primary lung carcinoma were studied. Of these, 17 were squamous cell carcinomas, 10 adenocarcinomas, nine small cell carcinomas, and one large cell carcinoma. The patients' ages ranged from 34-82 years. The male:female ratio was 3:6:1. Squamous cell carcinoma antigen was raised above the cutoff value of $1.5 \mathrm{ng} / \mathrm{ml}$ in $94.1 \%$ of squamous cell carcinomas, $20.0 \%$ of adenocarcinomas, and $11 \cdot 1 \%$ of small cell carcinomas. By comparison, CEA was raised above the cutoff value of $3.0 \mathrm{ng} / \mathrm{ml}$ in $70.6 \%$ of squamous cell carcinomas, $\mathbf{7 7 \cdot 8 \%}$ of small cell carcinomas, and $100 \%$ of adenocarcinomas. CEA and squamous cell carcinoma antigen were not raised in the patient with large cell carcinoma and in 14 healthy volunteers. None of 15 patients with a variety of benign lung diseases showed a rise of CEA, while two patients-a 25 year old Indian woman with pneumonia and a 64 year old Malay man with bronchial asthma-had raised squamous cell carcinoma antigen values above the cutoff. Serum CEA and squamous cell carcinoma antigen values did not seem to correlate with stage or degree of differentiation of the tumours.

Conclusions-The findings suggest that CEA is a good general marker for carcinoma, particularly adenocarcinoma. In contrast, squamous cell carcinoma antigen is more specific for squamous carcinoma.

$(\mathcal{F}$ Clin Pathol 1994;47:535-537)
Derived from TA-4 antigen, squamous cell carcinoma (SCC) related antigen has been successfully used as a marker for carcinoma of the uterine cervix. ${ }^{12}$ First described in 1977 by Kato and Torigoe, TA-4 antigen, a glycoprotein with a molecular weight of 48000 daltons, was purified from squamous cell carcinoma of the uterine cervix. ${ }^{3}$ Although most commonly associated with cervical squamous cell carcinoma, a rise in serum SCC antigen has also been noted in glandular epithelial malignancies of the cervix ${ }^{4}$ and other epithelial malignancies, including carcinomas of the head and neck, oesophagus and lung. ${ }^{5-7}$ Furthermore, in the cervix the concentration of SCC antigen has been noted to correlate with tumour bulk, stage, and extent of disease. ${ }^{18}$

Carcinoembryonic antigen (CEA) is one of the most commonly used serological markers for primary carcinoma of the lung. ${ }^{9}$ Various rates of detection, ranging from $50 \%$ to $80 \%$, have been reported. ${ }^{10-12} \mathrm{~A}$ prospective study was begun at University Hospital, Kuala Lumpur, to assess (1) the detection rate of primary carcinoma of the lung using the conventional CEA seroassay and (2) whether assaying serum SCC antigen before treatment in addition to CEA improves diagnostic sensitivity.

\section{Methods}

Patients were selected from those seen at the medical unit between August 1991 and October 1993. All patients clinically suspected of having primary carcinoma of the lung were assayed for SCC antigen (Abbott IMx) and CEA (Abbott IMx) using the microparticle enzyme immunoassay method before treatment. Of these, only histologically confirmed cases were considered for the study. Cases with recurrent carcinoma were not considered. Histological sections of all biopsy specimens were reviewed and classified according to the WHO histological classification system (1981). Histochemical and immunoperoxidase stains were carried out whenever necessary. Only histologically reconfirmed cases were finally included in the study. The tumours were graded as well, moderately, or poorly differentiated, and staged by the New International Staging System for Lung Cancer. ${ }^{13}$ Fifteen consecutive cases of clinically diagnosed benign lung lesions seen at the medical unit during the same period and 14 healthy volunteers were 
also seroassayed for CEA and SCC antigen. Arbitrary cutoff values of $1.5 \mathrm{ng} / \mathrm{ml}$ and 3.0 $\mathrm{ng} / \mathrm{ml}$ were adopted for SCC antigen and CEA, respectively.

\section{Results}

Eighty two cases of suspected primary lung carcinoma were seroassayed for SCC antigen and CEA during the study period. Thirty seven histologically re-confirmed cases were included in the study: 27 Chinese, seven Malay, and three Indian people (age range 34-82; mean 60.4 years). The number of men totalled 29 . Of the 37 cases, 17 were squamous cell carcinomas, 10 adenocarcinomas, nine small cell carcinomas and one large cell carcinoma. At the cutoff value of $1.5 \mathrm{ng} / \mathrm{ml}$, the SCC antigen concentration before treatment was raised in $16(94 \cdot 1 \%)$ squamous cell carcinomas, two $(20.0 \%)$ adenocarcinomas, and one $(11 \cdot 1 \%)$ small cell carcinoma. By comparison, $12(70 \cdot 6 \%)$ squamous cell carcinomas, seven $(77 \cdot 8 \%)$ small cell carcinomas, and all (100\%) adenocarcinomas showed increased CEA beyond the cutoff of 3.0 $\mathrm{ng} / \mathrm{ml}$. Both SCC antigen and CEA were not raised in the case of large cell carcinoma. Taking into consideration all the histological types of primary lung carcinoma, the serum SCC antigen concentration before treatment was raised in $51.4 \%$ of cases and that of CEA in $78.4 \%$ (table). While CEA detected 29 of the 37 cases, four cases of squamous cell carcinoma exhibited increased SCC antigen without a raised CEA. By seroassaying SCC antigen in addition to CEA, detection sensitivity was therefore increased from $78.4 \%$ to $89 \cdot 2 \%$.

The cases of benign lung diseases seroassayed included five cases of pneumonia, one of pulmonary tuberculosis, two of bronchiectasis, one of bronchial asthma, one of eosinophilic pneumonitis, two of chronic obstructive airway disease, one of fibrosing alveolitis, one of inflammatory pseudotumour, and one of spontaneous pneumothorax. CEA was not increased in any of these. SCC antigen concentrations were below the cutoff in 13 of these cases but raised to $6 \cdot 1 \mathrm{ng} / \mathrm{ml}$ in a 25 year old Indian woman with pneumonia, and marginally raised $(1.6$ $\mathrm{ng} / \mathrm{ml}$ ) in a 64 year old Malay man with bronchial asthma. Both serum CEA and SCC antigen were within normal limits in the 14 healthy controls.

Table Seropositivity for SCC antigen and CEA before biopsy in different histological types of primary lung carcinoma $(n=37)$

\begin{tabular}{|c|c|c|}
\hline $\begin{array}{l}\text { Histological } \\
\text { type }\end{array}$ & $\begin{array}{l}\text { SCC antigen } \\
\text { No }(\%) \text { positive }\end{array}$ & $\begin{array}{l}C E A \\
\text { No (\%) positive }\end{array}$ \\
\hline $\begin{array}{l}\text { Squamous cell } \\
\text { carcinoma }(n=17)\end{array}$ & $16(94 \cdot 1)$ & $12(70 \cdot 6)$ \\
\hline $\begin{array}{l}\text { Adenocarcinoma } \\
(n=10)\end{array}$ & $2(20 \cdot 0)$ & $10(100 \cdot 0)$ \\
\hline $\begin{array}{c}\text { Small cell carcinoma } \\
(\mathrm{n}=9)\end{array}$ & $1(11 \cdot 1)$ & $7(77 \cdot 8)$ \\
\hline $\begin{array}{l}\text { Large cell carcinoma } \\
(n=1) \\
\text { Total }\end{array}$ & $\begin{array}{l}0(0) \\
19(51 \cdot 4)\end{array}$ & $\begin{array}{l}0(0) \\
29(78 \cdot 4)\end{array}$ \\
\hline
\end{tabular}

A range of tumour differentiation and clinical stages were seen for each histological type of primary lung carcinoma. However, serum CEA and SCC antigen values correlated poorly with both degree of tumour differentiation and stage.

\section{Discussion}

The age range (34-82 years) and predominance of men among the cases noted here do not differ from other established studies. The male:female ratio of $3 \cdot 6: 1$ is similar to that in the United Kingdom (4.36:1) and United States of America (4:1). ${ }^{14}$ In this study $73 \%$ of the patients were Chinese. By comparison, the Chinese constituted only $45 \cdot 8 \%$ of all cases admitted to the medical unit during this period. There was a slight preponderance of Chinese patients, but the limited number of cases precludes meaningful statistical analysis. Whether primary carcinoma of the lung is more common in the Chinese is unclear and requires further study.

The detection rate of primary carcinoma of the lung by serological assay of CEA was $78.4 \%$, similar to that seen in other studies. ${ }^{10-12}$ As has been observed by other workers, ${ }^{1516}$ CEA was most efficient in the detection of adenocarcinomas, picking up all 10 cases. In contrast, CEA was raised in only $70.6 \%$ of squamous cell carcinomas and $77.8 \%$ of small cell carcinomas. Increased SCC antigen values were detected in $94.1 \%$ of squamous cell carcinomas while only $20.0 \%$ of adenocarcinomas and $11.1 \%$ of small cell carcinomas showed a similar rise. CEA seems to be a good marker for adenocarcinomas and SCC antigen for squamous cell carcinomas. Although most studies have shown that neurone specific enolase (NSE) is the ideal indicator of small cell carcinoma, ${ }^{17} 18$ CEA also seems to be reasonably effective, while SCC antigen is not.

Used as a single general tumour marker for primary lung carcinoma, irrespective of the histological type, serum SCC antigen managed to detect only $51.4 \%$ of cases. It seems to be inferior to CEA which detected $78.4 \%$ of the cases. However, the validity of this observation is debatable following the earlier observation that a rise in both CEA and SCC antigen is closely associated with histological type. None of the patients with benign pulmonary disease had increased serum CEA, while serum SCC antigen was raised in a patient with pneumonia and another with bronchial asthma. These findings are not unexpected as benign skin lesions and impaired renal function can result in raised SCC antigen. ${ }^{719}$ The reason for the rise in the two patients, whether due to the intrinsic lung disease or other independent conditions, is, however, not immediately apparent. Although not shown in this study, CEA is also known to rise in some benign lung conditions, false positive results being reported in as many as $10 \cdot 7 \%$ of cases. ${ }^{9}$

Like Upham and Campbell, ${ }^{7}$ we too found that SCC antigen values correlated poorly 
with both tumour stage and degree of differentiation. Similarly, CEA values did not correlate with either of the above parameters. This suggests that the serum concentrations of SCC antigen and CEA in primary lung carcinomas are influenced by factors not evaluated in this study. The possibility also remains that in lung cancers each case has its own baseline value of serological expression of these markers.

In conclusion, the findings of this study show that the sensitivity of CEA or SCC antigen is dependent on the histological type of lung cancer. CEA seems to be a more versatile general tumour marker that can detect squamous cell carcinomas, adenocarcinomas, and small cell carcinomas, although at varying degrees of sensitivity. None the less SCC antigen seems to be a better indicator for squamous malignancies. The seroassaying of SCC antigens in addition to CEA improved detection rate by $10 \cdot 8 \%$, from $78.4 \%$ to $89 \cdot 2 \%$. Therefore, for as long as squamous cell carcinoma continues to be a major histological type of primary lung cancer, we recommend seroassay of SCC antigen in addition to CEA to improve diagnostic accuracy. As a final note, it should be remembered that raised serum CEA and SCC antigen values should alert the physician to the possibility of malignancy but are not in themselves diagnostic. False positive results have always to be considered in the interpretation of results.

We are grateful to $\mathrm{CH} \mathrm{Ng}$ for technical assistance. This study was supported by the Malaysian Ministry of Science,

1 Maiman M, Feuer G, Fruchter RG, Shaw N, Boyce J. Value of squamous cell carcinoma antigen levels in invasive cervical carcinoma. Gynecol Oncol 1989;34:312-6.
2 Cheah PL, Yap SF, Looi LM, Sivanesaratnam V. Squamous cell carcinoma related antigen in uterine cervical carcinoma. Malaysian $\mathcal{f}$ Pathol 1991;13:37-41.

3 Kato $\mathrm{H}$, Torigoe T. Radioimmunoassay for tumor antigen of human cervical squamous cell carcinoma. Cancer 1977;40:1621-8.

4 Neuteufel W, Tatra G, Bieglmayer C. Serum squamous cell carcinoma antigen levels in women with neoplasms of the lower genital tract and in healthy controls. Arch Gynecol Obstet 1989;246:243-50.

5 Yamanaka N, Himi T, Harabuchi Y, Hoki K, Kataura A. Soluble immune complexes and squamous cell carcinoma related antigens in patients with head and neck cancer. Cancer 1988;62:1932-8.

6 Damle SR. Usefulness of squamous cell carcinoma antigen (SCC) in carcinoma of the esophagus. Clin Chem 1988; 34:1299-300.

7 Upham J, Campbell B. Utility of squamous cell carcinoma antigen (SCC Ag) as a tumour marker in pulmonary antigen (SCC Ag) as a tumour marker

8 Yazigi R, Munoz AK, Richardson B, Risser R. Correlation of squamous cell carcinoma antigen levels and treatment response in cervical cancer. Gynecol Oncol 1991;41. response.

9 Leichtweis B, Ebert W. Diagnostic value of new mucinmarkers CA M26 and MCA in comparison with carcinoembryonic antigen in lung cancer. Diagn Oncol 1992; 2:1-8.

10 Poulakis N, Sarandakou A, Rizos D, Phocas I, Kontozoglou T, Polyzogopoulos D. Soluble interleukin-2 receptors and other markers in primary lung cancer. Cancer 1991;68:1045-9.

11 Chevinsky AH. CEA in tumors of other than colorectal origin. Semin Surg Oncol 1991;7:162-6.

12 Niklinski J, Furman M, Laudanski J, Palynyczko Z, Welk $M$. Evaluation of carcinoembryonic antigen (CEA) and brain-type creatine kinase (CK-BB) in serum from patients with carcinoma of the lung. Neoplasma 1991;38: 129-35.

13 Mountain CF. A new international staging system for lung cancer. Chest 1986;89(Suppl):225S-33S.

14 Spencer H. Pathology of the lung. 4th ed. Oxford Pergamon Press, 1985:866.

15 Leichtweis B, Ebert W. Diagnostic value of the new mucin markers CA M26 and MCA in comparison with carcinoembryonic antigen in lung cancer. Diagnost Oncol 1992; 2:1-8.

16 Shimabukuro Z. A clinical evaluation of the versatility of various tumor markers in diagnosing the primary carcivarious tumor markers in diagnosing the primary carcinoma of

17 Gasser RW, Denz H, Huber H. Tumor markers in bronchus cancer. Wien Klin Wochenschr 1989;101: 476-9.

18 Jorgensen LG, Hansen HH, Cooper EH. Neuron specific enolase, carcinoembryonic antigen and lactate dehydrogenase as indicators of disease activity in small cell carcinoma. Eur f Cancer Clin Oncol 1989;25:123-8.

19 Duk JM, van Voorst Vader PC, ten Hoor KA, Hollema H, Doeglas HM, de Bruijn HW. Elevated levels of squamous cell carcinoma antigen in patients with a benign disease of the skin. Cancer 1989;64:1652-6. 